\title{
Editorial
}

\section{El trabajador de la salud en tiempos de covid-19}

Para el trabajador de la salud, habrá un "antes" y un "después" del CoviD-19. La emergencia sanitaria expuso la fragilidad de los sistemas de salud y su falta de preparación para hacer frente a semejante crisis, y se hizo visible el papel primordial de los trabajadores de la salud para enfrentar este desafío.

Antes de la pandemia, en la mayoría de los países, tanto ricos como pobres, había deficiencias en el mercado laboral del área de la salud, como recortes de personal — en ocasiones debidos a pérdidas por emigración-, desequilibrios en la composición profesional, inequidad en la distribución geográfica, programas educativos que no estaban alineados con las necesidades de los servicios, y deficiencias en la administración, la regulación y el manejo. Al menos desde el año 2004, ha habido conocimiento de la crisis en el ámbito del recurso humano en salud [1,2] y, sin embargo, los gobernantes decidieron hacer caso omiso, aun sabiendo que sus países adquirieron el compromiso formal de una salud con cobertura universal. La pandemia del 2020 ha sido un llamado de atención y aseguró que nadie olvide que la capacidad y el desempeño de los sistemas de salud depende principalmente de sus trabajadores.

Desde el inicio de la crisis, los trabajadores de la salud de diferentes partes del mundo han mostrado una dedicación excepcional y una capacidad de adaptación a una situación sin precedentes. Los que están en la primera línea de batalla en las unidades de cuidado intensivo han sido los más visibles; pero aquellos que prestan servicios menos impresionantes, aunque esenciales, como el mantenimiento de los ambientes asistenciales libres de patógenos o el transporte de pacientes, también respondieron de manera admirable. Muchos pagaron un alto precio poniendo su salud y, en algunos casos, sus vidas en peligro. La población y las autoridades políticas los aplaudieron de manera virtual y literal. Esta es una forma de reconocimiento merecida y apreciada, pero lo que los trabajadores necesitan realmente es el abordaje de temas como las pesadas cargas laborales, la baja remuneración, las pocas posibilidades de ascenso y las difíciles condiciones laborales.

Ya hay señales de que el trabajo en salud pos-Covid va a ser diferente. Por ejemplo, las consultas virtuales han aumentado de modo significativo a medida que la crisis avanza, y los profesionales de la salud y los pacientes se han adaptado bien a esta nueva forma de interacción. Es posible que la teleconsulta y la telemedicina se vuelvan frecuentes para la prestación de servicios como consultas de seguimiento, orientación a pacientes sobre el manejo y monitoreo de la adherencia a la medicación. Esto implica la necesidad de capacitar a los profesionales de la salud, actuales y futuros, en el uso adecuado de estas herramientas. Es posible que aparezcan nuevos roles que asumir, relacionados con funciones que aún no están establecidas. Otro cambio puede resultar de la preferencia de los adultos mayores por evitar residencias geriátricas, las cuales son el epicentro de la pandemia en casi todos los países; por lo tanto, la demanda de cuidados domiciliarios aumentará y serán necesarios trabajadores entrenados específicamente para la prestación de dichos servicios.

La crisis también demostró que las definiciones rígidas de funciones no son adecuadas en este contexto. Se requiere la flexibilidad y el aprovechamiento de todo el potencial de los trabajadores en todas las categorías. Ya existe presión para que se revise la regulación sobre el alcance de las funciones en la práctica de las enfermeras, los farmacéuticos, entre otros, y la posibilidad de la expansión de las mismas, como ha ocurrido en otros países [3]. En consecuencia, la división de tareas entre las diferentes profesiones de la salud puede variar significativamente en el futuro cercano.

Los gobernantes y los líderes enfrentan ahora el desafío de decidir cómo asegurar que el trabajador de la salud de su país esté alineado con las necesidades de servicio futuras de la población. Para este fin, deberán tener una visión clara del sistema de salud futuro que desean y los valores fundamentales que guiarán su organización y funcionamiento. Por ejemplo, la búsqueda de la cobertura universal en salud implica el fortalecimiento de la equidad en el acceso y la protección al riesgo financiero, para abordar las inequidades considerables reveladas por la crisis. Hay equidad cuando toda la población tiene acceso a los profesionales de la salud, independientemente de su capacidad de pago y sin ningún tipo de discriminación (clase social, origen étnico, religión, orientación sexual, etc.). Equidad también significa tener acceso a los profesionales de la salud en función de la importancia de la necesidad, es decir, la misma calidad del 
servicio para el tipo de urgencia o gravedad del problema de salud. Por consiguiente, los servicios de atención primaria sólidos y accesibles, y el trabajo calificado son el camino más firme hacia la cobertura universal [4].

De manera más general, la educación y las condiciones laborales del trabajador de la salud del futuro necesitan transformarse, en respuesta a los cambios demográficos, epidemiológicos y tecnológicos, y a las expectativas y demandas de la población para el acceso a servicios de calidad. Además, los líderes necesitan comprender y tener en cuenta el comportamiento de los nuevos trabajadores en la elección del área laboral, al lugar para trabajar, a la carga laboral que están preparados para aceptar y a un mercado laboral global en aumento.

En el continente americano, muchos países han empezado a moverse en la dirección correcta como respuesta a la epidemia del COVID-19, por ejemplo, mejorando la remuneración y las condiciones laborales, incluyendo seguridad y protección en el trabajo, y el acceso a entrenamiento adicional, y facilitando la integración de los nuevos trabajadores al mercado laboral, principalmente en el nivel de atención primaria [5]. Iniciativas similares están teniendo lugar en el resto del mundo. Aunque esto debe ser elogiado, los gobiernos deben continuar el progreso en esta dirección. Su labor es trabajar hacia la construcción de una conformación más eficiente de la fuerza de trabajo, concretamente, mediante el balance de la proporción entre enfermeras y médicos, y promoviendo la colaboración entre los trabajadores de los diferentes niveles de atención. Se debe asegurar la calidad de la educación y la práctica, a través de mecanismos como la acreditación independiente y organizaciones como los consejos de profesionales. Para lograr esto, los gobernantes deben fortalecer su capacidad para regular, planificar y gestionar el personal de la salud, con una visión que asegure la prestación de un servicio que cumpla con las necesidades y las expectativas de la población.

Las probabilidades de lograrlo aumentarán si los líderes tienen acceso a la información de bases de datos confiables, como la plataforma de cuentas del personal de salud (National Health Workforce Accounts) de la Organización Mundial de la Salud [6], si hay suficiente apoyo de los principales inversionistas y si hay suficientes recursos disponibles, los cuales requieren del apoyo gubernamental. En los últimos años, se ha promovido una mayor inversión en el trabajador de la salud, argumentando que se si realiza de manera adecuada, esto no será simplemente un gasto, sino una inversión sólida [7].

Es probable que ahora que la crisis del CoviD-19 y las deficiencias en el trabajo en salud han amplificado una crisis económica global, los gobernantes sí escuchen este mensaje.

\author{
Gilles Dussault \\ Salud Global y Medicina Tropical \\ Instituto de Higiene y Medicina Tropical \\ Universidad Nova de Lisboa \\ Traducción: \\ Agencia de Traducción UdeA - Escuela de Idiomas, Universidad de Antioquia \\ También disponible en inglés
}

\title{
Referencias
}

1. Chen L. Evans T, Anand S, et al., Human resources for health: Overcoming the crisis. The Lancet; 2004:364(9449):1984-90. Dor: https://doi. org/10.1016/S0140-6736(04)17482-5

2. World Health Organization. The World Health Report 2006: Working together for Health. Geneva: World Health Organization; 2006.

3. Maier C. Nurse prescribing of medicines in 13 European countries. Hum Resour Health. 2019;17:95. Dor: https://doi.org/10.1186/s12960-0190429-6

4. Dussault G, Kawar R, et al. Building the primary health care workforce of the 21st century. Geneva: World Health Organization [internet]; 2018. Disponible en: https://apps.who.int/iris/bitstream/handle/10665/328072/WHO-HIS-SDS-2018.48-eng.pdf?sequence=1\&isAllowed=y

5. Organización Panamericana de la Salud. Experiencias para destacar en gestión de recursos humanos para la salud en países de América del Sur en el contexto de Covid-19 [internet]; 2019. Disponible en: https://www.paho.org/es/noticias/27-5-2020-experiencias-para-destacar-gestionrecursos-humanos-para-salud-paises-america

6. World Health Organization. National health workforce accounts: Implementation guide [internet]; 2018. Disponible en: https://apps.who.int/ iris/handle/10665/275473

7. World Health Organization. High-Level Commission on Health Employment and Economic Growth [internet]; 2016. Disponible en: https:// www.who.int/hrh/com-heeg/en/ 